\title{
Systematic and functional analysis of horizontal gene transfer events in diatoms.
}

\author{
Emmelien Vancaester ${ }^{1,2}$, Thomas Depuydt ${ }^{1,2}$, Cristina Maria Osuna-Cruz ${ }^{1,2}$, Klaas Vandepoele ${ }^{1,2,3, *}$ \\ ${ }^{1}$ Department of Plant Biotechnology and Bioinformatics, Ghent University, Technologiepark 71, 9052 \\ Ghent, Belgium \\ ${ }^{2}$ VIB Center for Plant Systems Biology, Technologiepark 71, 9052 Ghent, Belgium \\ ${ }^{3}$ Bioinformatics Institute Ghent, Ghent University, Technologiepark 71, 9052 Ghent, Belgium \\ ${ }^{*}$ Corresponding author
}

\section{Abstract}

Diatoms are a diverse group of mainly photosynthetic algae, responsible for $20 \%$ of worldwide oxygen production, which can rapidly respond to favourable conditions and often outcompete other phytoplankton. We investigated the contribution of horizontal gene transfer (HGT) to its ecological success. A systematic phylogeny-based bacterial HGT detection procedure across nine sequenced diatoms showed that 3-5\% of their proteome has a horizontal origin and a large influx occurred at the ancestor of diatoms. More than $90 \%$ of HGT genes are expressed, and species-specific HGT genes in Phaeodactylum tricornutum undergo strong purifying selection. They are implicated in several processes including environmental sensing, and expand the metabolic toolbox. Cobalamin (vitamin B12) is an essential cofactor for roughly half of the diatoms and is only produced by bacteria. Genes involved in its final synthesis were detected as HGT, including five consecutive enzymes in Fragilariopsis cylindrus. This might give diatoms originating from the Southern Ocean, a region typically depleted in cobalamin, a competitive advantage. Overall, we show that HGT is a prevalent mechanism that is actively used in diatoms to expand its adaptive capabilities. 


\section{Introduction}

Horizontal, also dubbed lateral, gene transfer (HGT) is the transfer of genetic information between reproductively isolated species by a route other than direct exchange from parent to progeny. Although HGT events are widespread and well documented among prokaryotes, they are much rarer in eukaryotes. Nevertheless, recently several examples of HGT from archaea or bacteria into eukaryotes have been reported. Functional HGT events have been described for almost all unicellular eukaryotic lineages, including fungi ${ }^{1,2}$, extremophilic red algae ${ }^{3}$, green algae ${ }^{4}$, rumen-associated ciliates $^{5}$, oomycetes ${ }^{6}$ and photosynthetic diatoms ${ }^{7,8}$. Next to events involving the maintenance of preexisting functions, which occur mainly in endosymbiotic relationships, innovative events have been described which provide the recipient with new functions or an altered phenotype ${ }^{9}$. Although the uptake of genetic material happens by chance, fixation does not, making HGT predominantly important in the following processes: i) the alteration of iron uptake and metabolism ${ }^{8,10,11}$, ii) adaptation to an anaerobic lifestyle ${ }^{12,13}$, iii) nucleotide import and synthesis ${ }^{1,14}$, iv) novel defence mechanisms ${ }^{15,16}$, v) mechanisms to cope with stressors such as salt ${ }^{17,18}$, temperature ${ }^{4}$ and heavy-metal concentrations ${ }^{3}$ and vi) expansion of its metabolic capacities ${ }^{2,5,6}$.

Diatoms (Bacillariophyta) are one of the most abundant and species-rich groups of phytoplankton and release between $20-25 \%$ of the global amount of oxygen ${ }^{19}$. They can rapidly adapt to local conditions, outcompete other photosynthetic eukaryotes and dominate oceanic spring blooms, as long as silicon is not limited ${ }^{20}$. Moreover, they are found throughout every aquatic photic zone of this planet, such as oceans, intertidal zones, freshwater bodies, soil and even ice ecosystems ${ }^{21}$. Molecular clock evidence suggests that diatoms emerged between 225 and 200 million years ago ${ }^{22}$ and their origin may be related to the end-Permian mass extinction which occurred around 250 million years ago. In the early Cretaceous, between 150 and 130 million years ago, diatoms split into the centric and pennate lineage. Several whole-genome sequences of representatives from polar centrics (Thalassiosira pseudonana ${ }^{23}$, Thalassiosira oceanica ${ }^{24}$, Cyclotella cryptica ${ }^{25}$ ), araphid pennates (Synedra acus ${ }^{26}$ ) and raphid pennates (Phaeodactylum tricornutum ${ }^{7,27}$, Fistulifera solaris ${ }^{28}$, Fragilariopsis cylindrus ${ }^{29}$, Pseudo-nitzschia multistriata $^{30}$ ) have become available in recent years, which allows the analysis of the evolutionary history within diatoms. It is not fully understood how HGT has contributed to the ecological success of this environmentally important group of organisms. Moreover, diatoms evolved from several endosymbiotic events and their plastid is thought to have originated from a red alga, which has also contributed to their genetic set-up.

Although HGT detection has been previously performed in diatoms within the context of genome projects $^{7,24,25,27,30}$, they were based on different methodologies and criteria and are therefore not directly comparable. While some studies used phylogenetics ${ }^{7,30}$, others relied purely on sequence homology searches ${ }^{24,25,27}$. In this study, we sought to systematically detect HGT events simultaneously across all sequenced diatoms. We delineated genes from horizontal descent using a high-throughput gene family phylogenetics-based approach, which allows dating transfer events. Here, we comprehensively explore the functional bias of HGT genes in diatoms and for the first time gain insight into their expression dynamics and patterns of selection. 


\section{Results}

\section{Detection and phylogenetic distribution of diatom HGT candidates}

Twenty unicellular eukaryotic species (Table S1) were selected to deduce the contribution of bacterialderived HGT. The identification of bacterial-to-eukaryotic HGT was achieved by building a phylogenetic tree per gene family. Therefore, all protein-coding genes from 20 eukaryotic species (Figure 1a) were clustered in 145,601 gene families, of which $32 \%$ are genes lacking similarity to any other protein in this dataset, followed by phylogenetic tree construction for 8,476 gene families having similarity to bacterial proteins. Also the species topology of these 20 unicellular eukaryotes was constructed, both based on single-locus trees and a concatenation-based approach of 156 near-single copy gene families (138,948 amino acids) (Figure 1a), with the haptophyte Emiliania huxleyi as an outgroup. Having the species tree available, allows for the dating of HGT candidates.

To avoid the misclassification of contaminating DNA present in the genome assembly as genomic regions originating by HGT, several quality analyses were performed. The guidelines proposed by Richards and Monier ${ }^{31}$ were followed to exclude incorrect inference of HGT. Therefore, the gene origin was determined by phylogenetic tree construction followed by inspection of species-specific HGT genes. Also the percentage GC and the integration of HGT genes across chromosomes was assessed. First, the fraction of species-specific HGT was compared among all diatoms. More than $75 \%$ $(2146 / 2844)$ of the predicted HGT genes in S. acus were only detected in this genome, while in all other species this fraction was drastically lower $(11.58+/-9.25 \%)$ (Figure $1 \mathrm{~b})$. A donor analysis of these genes revealed that many were derived from Sphingomonas $s p$., which has been described to be associated with $S$. acus in culture ${ }^{32}$. Contigs flagged to be contaminant based on a nucleotide sequence similarity search against all available Sphingomonadales genomes were clearly separable from $S$. acus based on their significantly lower percentage GC (Figure 2 a) $\left(42.1 \%\right.$ vs $63.3 \%$, p-value $<2 \times 10^{-16}$ ). Therefore, all 695 nuclear contigs having a GC content above $50 \%$ were removed, reducing the nuclear $S$. acus genome size by $4 \mathrm{Mb}$ to $94.38 \mathrm{Mb}$ and retaining 23,719 genes. Interestingly, the HGT detection procedure succeeded in both flagging the contaminant as detecting HGT events in the $S$. acus genome (Figure 2b). Despite the fact that in several other diatoms the GC content was significantly different between genes from horizontal and vertical descent, the mean difference never exceeds two percentage points (Figure S1).

Next, the enrichment of HGTs per contig or chromosome was evaluated to assess whether certain regions are derived from contamination, yielding no clear examples of clustering of HGT genes on specific genomic locations. The distribution of HGTs across the chromosome-level genomes of P. tricornutum and T. pseudonana is plotted in Figure S2 and shows an unbiased distribution of HGT genes. As it has been proposed that the transfer of transposable elements could be associated with facilitating gene transfer ${ }^{33}$, the distance between every gene and its closest transposable element was calculated in $P$. tricornutum. Species-specific HGT genes were significantly closer to transposable elements (TEs) ( $p$-value $1.6 \times 10^{-03}$ ), while the same was also true for vertically descended speciesspecific genes ( $p$-value $2.7 \times 10^{-14}$ ). This suggests that novel genes are more likely to integrate and become fixed close to repetitive regions.

Except for a fraction of genes in the $S$. acus data set, we could not identify genomic properties indicating that the identified HGT genes are caused by contamination. In total, 7,461 diatom genes were defined as having HGT origin, covering 1,979 gene families. This reflects 509 to 1,741 genes per species, making 3 to 5 percent of the diatom gene repertoire predicted to be HGT (Figure 1a). This is similar to previous phylogenetic-based estimations of HGT content in diatoms, which ranged from 587 genes $(4.8 \%)$ in $P$. tricornutum ${ }^{7}$ to 438 in $P$. multistriata ${ }^{30}$ (3.6\%) and is slightly higher than what was 
reported in the anaerobic gut parasite Blastocystis hominis ${ }^{34}(2.5 \%)$, where next to bacterial HGT also other transfers were described. The lower frequency in this stramenopile could be due to its constrained and reduced genome size as a result of its parasitic lifestyle. On average, a HGT gene family consisted out of 3.76 diatom genes and 2.55 diatom species. In total, only 106 HGT families were present in all nine diatoms. For 69 gene families the HGT copies were significantly expanded in at least one species, of which notably 26 and 21 gene families were expanded respectively in S. robusta and $S$. acus (Table S2). Indeed, gene family expansion by duplication has been observed before following HGT integration in eukaryotes ${ }^{1,35}$ and this could be a strategy to diversify the original acquired function.

The age of all gene families of vertical descent was determined based on the lowest common ancestor of the observed species. Similarly, the most likely time point of integration for every HGT was determined using the species composition of the acceptor branch in the phylogenetic tree (Figure 1a). The large number of HGT gene families that can be attributed to the ancestor of diatoms is striking, ranging from $15 \%$ in S. robusta to $30 \%$ in T. pseudonana (Figure 1b). Another study ${ }^{27}$ also detected a continuous flux of genes from prokaryotes during the evolutionary history of $P$. tricornutum. However, they claimed that most influx occurred at ancestor of the photosynthetic Stramenopiles (Ochrophytes), while our results indicate this happened more recently in the ancestor of the diatom clade.

Finally, several structural gene features were evaluated according to their mode of inheritance. The coding gene length of vertically descended species-specific genes in all diatom species was significantly shorter compared to all other genes ( $p$-value $<2 \times 10^{-16}$ ) and significantly shorter to the species-specific HGT genes in all diatoms, except for T. pseudonana (Figure S3). In yeast, it has also been observed that de novo genes were on average shorter than conserved and horizontally transferred genes ${ }^{36}$. Speciesspecific HGT genes on the other hand, were significantly shorter to all other genes in C. cryptica ( $p$ value $2.1 \times 10^{-02}$ ) and $S$. robusta ( $p$-value $\left.1.4 \times 10^{-05}\right)$. Given that introns are a typical eukaryotic gene feature, HGT genes are expected to have a shorter total intron length, especially for recent acquisitions as HGT genes adapt to their recipient genome. The intron length of HGT genes was significantly shorter in several pennate diatoms (F. solaris: $1.8 \times 10^{-03}, P$. tricornutum: $3.4 \times 10^{-02}$, S. robusta: $1.1 \times 10^{-07}$ and $S$. acus: $4.6 \times 10^{-03}$ ) and for several diatoms the young species-specific HGT genes had shorter introns than the rest of the gene repertoire (F. cylindrus: $1.1 \times 10^{-03}$, F. solaris: $9.8 \times 10^{-03}, P$. tricornutum: $1.1 \times 10^{-02}, S$. robusta: $2 \times 10^{-09}$ and $C$. cryptica: $4.9 \times 10^{-02}$ ). These results indicate that introns become an emerging property of HGT genes after integration.

\section{The functional landscape of diatom HGT genes}

To gain insight in the functional repertoire of HGT genes, a gene ontology (GO) and functional domain (Interpro) enrichment was performed. Out of the 7,461 diatom HGT genes, 6,024 (81\%) were annotated with an Interpro domain and 3,893 (52\%) with a GO term. The only GO term which was enriched for HGT genes in all nine diatom species is pseudouridine synthesis (GO:0001522), while enriched protein domains covered pseudouridine synthase (IPR006145), S-adenosyl-L-methioninedependent methyltransferase (IPR029063) and nitroreductase (IPR029479). An overview of the enriched functional categories across different ages can be found in (Figure S4, Figure S5). A more indepth exploration of several functional categories is given in Supplementary Note 1, while an overview of all discussed functions and their corresponding gene families can be found in Table S3.

\section{Cobalamin uptake}

Cobalamin (vitamin B12) is a complex molecule composed out of a central cobalt-containing corrin ring, a lower ligand of 5,6-dimethylbenzimidizole (DMB) and an upper axial ligand that can either be an hydroxy-, cyano-, methyl or adenosyl group. Vitamin B12 acts as a coenzyme in three enzymes in eukaryotes: methylmalonyl-CoA mutase (MCM), type II ribonucleotide reductase (RNRII) and 
methionine synthase (METH). Despite that more than half of the algal species surveyed $(171 / 326)^{37}$ are auxotrophic for vitamin B12, including 37 out of 58 diatoms, de novo synthesis has only been described to occur in prokaryotes. Therefore cobalamin availability alters the composition of marine phytoplankton communities ${ }^{38}$. The exchange of cobalamin in return for organic compounds is believed to underpin the close mutualistic interactions between heterotrophic bacteria and auxotrophic algae ${ }^{39}$ A correlation was detected between the scattered phylogenetic pattern of absence of a cobalaminindependent methionine synthase (METE) and auxotrophy for this vitamin ${ }^{40}$. It has been suggested that this loss has a biogeographical basis as there is a tendency for diatoms occurring in the Southern Ocean to retain METE more often ${ }^{41}$. Moreover, it has been recently proven that cyanobacteria produce the chemical variant pseudo-cobalamin, where adenine substitutes DMB as the lower ligand, which is less bioavailable to eukaryotic algae ${ }^{42}$. However, some species, including $P$. tricornutum and $E$. huxleyi ${ }^{43}$ can remodel this to cobalamin using CobT, CobS and CobC via the nucleotide loop assembly ${ }^{39,42}$. Here BluB, necessary for $\mathrm{DMB}$ production ${ }^{44}$, was detected to have originated by HGT from alphaproteobacteria in F. cylindrus, $P$. multistriata and $P$. multiseries (Figure 3a). More than 90 percent of the cobalamin-producing alpha- and gammaproteobacteria encode BluB ${ }^{39}$. Moreover, five HGT genes were detected in the final synthesis of the cobalamin biosynthesis pathway, which can also function as scavenging and repair genes: $\mathrm{CobN}, \mathrm{CobA} / \mathrm{CobO}, \mathrm{CobO} / \mathrm{CbiP}, \mathrm{CobD} / \mathrm{CbiB}$ and CobU/CobP (Figure 3a). These genes were previously also detected in diatom metatranscriptomes and $P$. granii ${ }^{45,46}$, where CobN, CobS and CobU were more highly expressed under iron replete conditions. Interestingly, for all diatoms, except for $C$. cryptica, the CbiB gene also contains the CbiZ domain, which is involved in the removal of the lower ligand ${ }^{43}$. Only $F$. cylindrus and $P$. multiseries contain the full suite of these detected HGT genes in their cobalamin pathway, while $P$. tricornutum, $F$. solaris and $S$. acus possess none (Figure 3a). Interestingly, while $P$. multiseries is auxotrophic for cobalamin, F. cylindrus is not. Thus, despite the presence of the cobalamin independent methionine synthase METE, F. cylindrus expanded its repertoire of cobalamin synthesis genes and prefers to maximally optimize its uptake to perform methionine synthesis by the more efficient METH. By querying the metatranscriptomic TARA Oceans data it was clear that $\mathrm{CobU}, \mathrm{CbiZ}+\mathrm{CbiB}$ and $\mathrm{CobQ}$ are significantly correlated with nitrate concentration and day length (Figure 3b) (Figure S6,Figure S7), while CobU and CbiZ+CbiB are anticorrelated with temperature (Figure S8) and CobU and CobQ are anti-correlated with iron (Figure S9). HGT genes in the cobalamin pathway are particularly abundant in the Southern and Pacific Ocean (Figure $3 \mathrm{~b}$ ). The lower production rate of bacteria in low temperature and the photodegradation of cobalamins, which could be of particular importance during arctic summers, might explain the cobalamin limitation and the specific expression of vitamin B12-related genes in these regions of the ocean.

\section{Environmental adaptation to light sensing and cold protection}

Diatoms employ photosensory proteins to gain information about their environment and respond to changing light conditions. Proteorhodopsins (PR) perceive light to drive ATP generation and are especially important when photosynthesis is comprised during iron-limiting conditions. This study confirms the bacterial origin of the PR-genes in F. cylindrus and Pseudo-nitzschia granii ${ }^{47}$, next to brown algae, dinophytes and haptophytes. Furthermore, the red/far-red light sensing phytochrome $\mathrm{DPH}{ }^{48}$ was detected as HGT in P. tricornutum (1 copy), S. robusta (4), S. acus (7), C. cryptica (1) and T. pseudonana (1), and formed together with brown algae an independent branch from green algal and fungal DPHs, similar as in previous reports ${ }^{48,49}$ and was predicted to have originated from HGT.

Arctic diatoms such as F. cylindrus undergo periods of prolonged darkness, low temperature and high salinity. Their ability to thrive in these conditions could be partially attributed to cryoprotectants that interfere with the growth of ice ${ }^{50}$. Ice-binding proteins were found to be laterally transferred from a 
basidiomycete lineage to Fragilariopsis curta and F. cylindrus ${ }^{51}$. Also the phylogenetic tree inferred in this study detected relatedness between fungal and diatom antifreeze proteins, but wasn't classified as HGT as this pipeline only detects bacterial-to-eukaryotic HGT. However, a second gene family of $F$. cylindrus proteins containing the ice-binding protein domain (IPR021884), was found to be transferred from Cryobacterium.

\section{Carbon and nitrogen metabolism}

Diatoms can rapidly recover from prolonged nitrogen limitation due to presence of the urea cycle that allows for carbon fixation into nitrogenous compounds ${ }^{52}$. Two genes in the metabolic branches derived from this pathway, carbamate kinase and ornithine cyclodeaminase were found to be laterally transferred, both here as in previous studies ${ }^{7,52}$. The latter enzyme is responsible for the conversion of ornithine to proline, which is the main osmolyte during salt stress in diatoms. Another way of a nitrogen storage and translocation is the catabolism of purines to urate that can be further degraded to allantoin. It was found that plants and diatoms independently evolved a fusion protein (Urah-Urad domain; allantoin synthase) to perform the second and third step in this urate degradation pathway ${ }^{53}$. Exactly as $\mathrm{in}^{53}$, this gene was detected to be laterally transferred from alphaproteobacteria, where this fusion event occurred, to the ancestor of haptophytes and stramenopiles.

Moreover, several genes in carbohydrate metabolism were found to be laterally transferred. The acetyl-CoA conversion to acetate occurs in a two-step process where phosphate acetyltransferase (PTA) adds a phosphate group to form acetylphosphate, that is in turn is catalyzed to acetate by acetate kinase $(A C K)^{54}$. The PTA gene family was found to have bacterial origins and emerged in the ancestor of haptophytes and stramenopiles. In all diatoms, except for F. cylindrus and P. multistriata, multiple copies were found of this gene. Also acetate kinase was detected as a HGT gene in the pennate diatoms $P$. tricornutum, S. robusta and S. acus. Furthermore, this enzyme was predicted to be involved in the bifid shunt ${ }^{54}$. Here, the key enzyme XPK cleaves xylulose-5-phosphate to acetyl-phosphate and glyceraldehyde-3-phosphate, followed by conversion of acetyl-phosphate to acetate by ACK. Also XPK was laterally transferred in the pennate diatoms, single-copy in $P$. tricornutum and significantly expanded to five copies in S. acus. XPK and ACK are syntenic in P. tricornutum, what was already suggested to point to a bacterial origin as this spatial organization is also detected in Proteobacteria and Cyanobacteria ${ }^{54}$. Interestingly, S. acus has also conserved the physical association of XPK and ACK and maintained a bidirectional promoter, although an inversion of the gene order occurred (Figure S10). Furthermore, phosphofructokinase and the cytosolic fructose-bisphosphate aldolase Fba4 in the glycolysis ${ }^{55}$, phosphopentose epimerase ${ }^{56}$ in the pentose phosphate pathway and a putative D-lactate dehydrogenase are enzymes that were predicted to be transferred from bacteria present in diatoms. Finally, also bacterial xylanases, glucanases and glucosidases expanded the carbohydrate metabolic repertoire in diatoms.

The biosynthetic aspartate-derived pathway to synthesize the four amino acids, lysine, threonine, methionine and isoleucine was completed due to $\mathrm{HGT}^{57}$. Aspartate semialdehyde dehydrogenase (asd) performs the second step in this pathway and is derived from Proteobacteria. The end product Laspartate 4-semialdehyde can either be used by dihydrodipicolinate synthase (dapA) towards lysine biosynthesis, or by homoserine dehydrogenase (thrA) towards threonine and methionine. Both genes were laterally transferred from bacteria. The metabolic pathways of other amino acids were also affected, the last step in tryptophan synthesis is achieved by tryptophan synthase. While in diatoms the alpha and beta subunit of this enzyme are merged, in P. tricornutum an extra copy of the beta subunit is present ${ }^{58}$ (Phatr3_J52286) that was deemed bacterial. Also alanine racemase, arginine biosynthesis ArgJ, leucyl-tRNA synthetase leuRS2, glycyl-tRNA synthetase glyRS2 and tyrosine-tRNA ligase tyrRS2 were laterally transferred. 
Genomic sequence information from ten Phaeodactylum accessions, belonging to four clades sampled across the world ${ }^{59}$, was used to do determine the maintenance and selection pattern across the detected HGT genes. The retention of species-specific HGT genes across different strains confirmed their horizontally derived origin and did not point to contamination (for more details, see Supplementary Note 2). Moreover, analyzing gene selection patterns gives an indication on the strength of functional conservation. Variant calling resulted in a data set of 585,715 high-confidence bi-allelic SNPs. The total number of SNPs per strain across the genome was low and ranged from $0.96 \%$ to $1.37 \%$ (Table S4). To detect selective pressure, $\pi \mathrm{N} / \pi \mathrm{S}$, was calculated. This metric compares the fraction of synonymous and nonsynonymous mutations within a coding open-reading frame across strains. A gene experiencing neutral evolution has a $\pi \mathrm{N} / \pi \mathrm{S}$ value of 1 , whilst a value smaller than 1 signifies negative purifying selection. The smaller the ratio of non-synonymous and synonymous nucleotide diversity, the stronger is the level of purifying selection acting on the gene. The average synonymous nucleotide diversity $(\pi \mathrm{S})$ across all accessions is 0.009 , while the non-synonymous nucleotide diversity $(\pi N)$ is 0.003 , thus the genome-wide average $\pi N / \pi S$ ratio is 0.3 . This value is similar to what was described in Rastogi et al..$^{59}$ and means most genes undergo strong purifying selection. The average $\pi N / \pi S$ for genes of vertical descent is 0.302 , while for HGT genes it is significantly lower at $0.268\left(p\right.$-value $\left.5.9 \times 10^{-4}\right)$. When comparing the $\pi \mathrm{N} / \pi S$ ratios for HGT and vertical genes across age classes (Figure 4), it is apparent that this difference is due to the youngest gene categories, being the Phaeodactylum-, raphid pennate diatoms and pennate diatoms specific genes, where vertical genes are less constrained than HGT genes in those age categories. To the best of our knowledge, this is the first time the selection pressure of bacterial HGT genes is assessed in unicellular eukaryotes and compared with vertically descended genes while taking age into account. Although it has already been observed that de novo genes display patterns of rapid evolution and the strength of purifying selection increases with $\mathrm{age}^{36}$, it is remarkable to observe that HGT genes deviate from this pattern. Unlike recent innovations from vertical descent, young HGT genes are quickly integrated in the biological network exemplified by their high levels of purifying selection.

\section{Expression and co-expression network analysis of HGT genes}

The availability of RNA sequencing experiments in several diatoms, allows for the construction of genome-wide gene expression atlases quantifying gene expression levels across a wide range of conditions. These compendia consisted out of 13 to 76 conditions per species, all having biological replicates per condition (Table S5). The vast majority of HGT genes are expressed: in P. tricornutum all HGT genes are expressed, in T. pseudonana 558 out of 580 (96\%), in S. robusta 1597 out of 1741 (92\%) and in F. cylindrus 741 out of 762 (97\%). Given that most HGT genes are kept under purifying selection in $P$. tricornutum and are transcribed in diatoms, this is indicative that they are functional and can play a vital role in expanding the functional repertoire. Indeed, $64 \%$ of the predicted HGT genes in $P$. tricornutum were translated into proteins in a proteogenomic analysis ${ }^{60}$. This is similar to $63 \%$ of all proteins in the genome that were detected to be translated.

Next, the expression specificity was calculated per gene, where a low value signifies broad expression in many (or even all) conditions and a value close to one indicates expression in one or a few. Speciesspecific genes have a higher mean condition-specific expression, both for vertical as horizontal derived genes in $P$. tricornutum ( $\mathrm{p}$-value $\left.<2 \times 10^{-16}, 1.1 \times 10^{-06}\right)$, S. robusta $\left(<2 \times 10^{-16}, 1.6 \times 10^{-13}\right)$, F. cylindrus $\left(<2 \times 10^{-}\right.$ $\left.{ }^{16}, 2.2 \times 10^{-03}\right)$ and $T$. pseudonana $\left(<2 \times 10^{-16}, 3.7 \times 10^{-02}\right)$. A declining trend of condition-specificity was observed over time. Whenever there was a significant difference in condition specificity between HGT and vertical genes within the same age category, HGT genes consistently displayed on average a more specific expression pattern (Figure 5). A high tissue-specificity for species-specific genes which 
decreases over time has also been observed in mouse ${ }^{61,62}$. Interestingly, the selection pressure in $P$. tricornutum across all age classes and per mode of inheritance is not correlated with expression specificity (Figure S11), showing that genes having a highly specific expression are not necessarily under less purifying selection, defying the trend that was previously observed in mammals ${ }^{63}$.

Based on a global co-expression $P$. tricornutum network constructed using an expression atlas comprising 211 samples, for every gene in $P$. tricornutum the co-expression neighbourhood was defined as a module. Subsequently, these modules and the known gene function for genes part of this module were used, through guilt-by-association analysis, to gain functional insights in the detected HGT genes. For 19 HGT genes the co-expression modules confirmed enrichment for at least one known function. Fructose-bisphosphate aldolase $\mathrm{Fba} 4$ is enriched in its co-expression module for genes involved in a carbohydrate metabolic process and aspartate semialdehyde dehydrogenase (asd) has enrichment for amino acid biosynthesis. New functions were attributed to 320 out of 509 HGT genes based on significant $\mathrm{GO}$ enrichment of the co-expression modules. For example, two HGT proteins involved in amino acid synthesis - tryptophan synthase $\beta$ chain ( $p$-value $\left.2.2 \times 10^{-16}\right)$ and ArgJ ( $p$-value $1 \times 10^{-15}$ ) - are predicted to be coregulated with photosynthetic genes, although both proteins do not contain the chloroplast targeting peptide. The co-expression neighbourhood of phosphofructokinase is significantly enriched ( $p$-value $3.7 \times 10^{-06}$ ) to be involved in the Krebs cycle, while this protein is part of the glycolysis and directly upstream of the citric acid cycle. Also Phatr3_J40382, which contains a pyruvate kinase-like domain, is enriched for this $\mathrm{GO}$ term, and this corroborates its metabolic function. Methionine sulfoxide reductase MsrB (Phatr3_J13757) was predicted to have a similar expression pattern to genes partaking in iron-sulphur cluster assembly ( $p$-value $\left.1.1 \times 10^{-03}\right)$ and metal ion transport ( $p$-value $\left.1.4 \times 10^{-02}\right)$. In yeast these proteins were already shown to have a protective role for FeS clusters during oxidative stress ${ }^{64}$. The far-red light phytochrome DPH1 is enriched for genes involved in transcriptional regulation ( $\mathrm{p}$-value $5.5 \times 10^{-03}$ ), which could point to its primary role in the light sensing cascade. These results demonstrate that co-expression network analysis offers a pragmatic means to predict the biological processes HGT genes are involved in.

\section{Discussion}

Through the application of phylogeny-based HGT detection, we identified 1,979 gene families with a horizontal origin in diatoms. Although HGT detection has been previously performed in diatoms, this is the first large-scale and systematic approach of HGT detection across all available sequenced diatoms. While some previous studies were based on phylogenetics ${ }^{7,30}$, most relied purely on sequence homology searches ${ }^{24,25,27}$, while it has been shown that the degree of gene similarity does not necessary necessarily reflect phylogenetic relationships ${ }^{65,66}$. Although HGT had been previously predicted in P. multistriata, C. cryptica, T. oceanica and twice in P. tricornutum, only a fraction of HGT genes were confirmed across these studies, going from 6 to $45 \%$ (Figure S12). This could be due to the usage of different methods, criteria and underlying databases. For example, horizontal genes were defined in T. oceanica if they didn't show high similarity with any other stramenopile and thus contain $T$. oceanica-specific genes from both bacterial- and eukaryotic-to eukaryotic origin. Nonetheless, the overlap between all methods is still significantly higher than expected by chance for all species.

Assessing the strength of purifying selection using genome-wide nucleotide diversity information showed that lateral gene transfer is quickly followed by fixation compared to the retention of new genes originating from non-coding regions, so-called de novo genes. Moreover, most species-specific HGT genes are present across all strains in $P$. tricornutum, suggesting they were acquired prior the divergence of these strains and are actively maintained in the population. This underlines the importance of HGT in diatoms and indicates that fixation of a laterally transferred gene takes place quickly after the initial uptake. Indeed, in grasses it was recently shown that several plant-to-plant LGT 
275 fragments were rapidly integrated and spread across the population, after which erosion occurred on

276 neutrally selected genes within those fragments ${ }^{67}$.

277 Among the HGT events, we detected the transfer of five concurrent genes of the vitamin B12

278 biosynthetic pathway. A cobalamin addition experiment in a high-nutrient low chlorophyll (HNLC)

279 region in the Gulf of Alaska significantly altered the species composition, going from diatom-

280 dominated plankton to an increased fraction of ciliates and dinoflagellates ${ }^{68}$. This could be explained

281 by the presence of these HGT genes and the corresponding enhanced uptake mechanism of vitamin

282 B12 and its analogues, which give diatoms a competitive advantage during limiting conditions. In

283 conclusion, our results support a high genetic plasticity and ability for local adaptation in diatoms due

284 to HGT. 


\section{Figure legends}

Figure 1: Overview of HGT events across diatoms. a, Species phylogeny determined by IQ-Tree with values at the internal nodes that denote bootstrap, gene- and site-concordance factors respectively. Branches are coloured according to their phylogenetic classification. Bold and framed values at internal nodes reflect the number of predicted HGT events. For diatoms, the number of species-specific GFs of HGT origin, the total number of HGT genes and its fraction of the proteome to be originating from HGT is tabularised. For S. acus the number of HGT genes both prior and after removal of contamination is mentioned. $\mathbf{b}$, Distribution of the age classes of HGTs across the nine investigated diatom species.

Figure 2: Contamination of Sphingomonadales in genome of $S$. acus. a, Percentage GC of $S$. acus contigs versus nucleotide sequence similarity to Sphingomonadales based on at least $70 \%$ identity and $25 \%$ alignment coverage. $\mathbf{b}$, Percentage GC of $S$. acus contigs versus presence of a HGT candidate within each contig.

Figure 3: Cobalamin pathway in diatoms. a, Overview of the cobalamin biosynthesis pathway and its presence in diatoms. Genes in bold are of horizontal descent and the presence of a gene is displayed by a filled circle, diamond or square depending on its position in the pathway. $\mathbf{b}$, Expressed number of diatom sequences of several HGT genes involved in the cobalamin pathway across stations sampled worldwide during the TARA Oceans project in the surface layer, coloured according to their nitrate concentration.

Figure 4: Selective pressure over time in $\boldsymbol{P}$. tricornutum. Distribution of selective pressure, measured by $\pi \mathrm{N} / \pi \mathrm{S}$, across age classes sorted from young to old and per origin in P. tricornutum. Number of genes is indicated in between brackets. The asterisks denote a statistical difference per type within the same age category and have the following confidence range for $p$-values; ${ }^{*}: 0.05,{ }^{* *}: 0.01,{ }^{* * *}: 0.001$, $* * * *: 0.0001$.

Figure 5: Expression specificity over time in diatoms. Distribution of expression specificity across age classes sorted from young to old and per origin in four diatoms. The number of genes is indicated in between brackets. The asterisks denote a statistical difference per type within the same age category and have the following confidence range for $\mathrm{p}$-values; ${ }^{*}: 0.05,{ }^{* *}: 0.01,{ }^{* * *}: 0.001,{ }^{* * * *}: 0.0001$. 


\section{Material and methods Gene family construction}

The publicly available genomes of 17 stramenopiles, 1 alveolate, 1 rhizarian and 1 haptophyte (listed in Table S1) were downloaded and their nuclear proteomes, totalling to 398,001 protein-coding genes, were searched for similarity in an all-against-all fashion with BLASTp (version 2.6+) using an e-value cut-off of $10^{-5}$ and retaining maximum 4,000 hits. Next, clustering of these protein-coding genes was performed using OrthoFinder (version 2.1.2) ${ }^{69}$.

\section{Species tree phylogeny}

To delineate the species phylogeny for all SAR members, using Emiliania huxleyi as an outgroup, OrthoFinder gene families where all species have a copy number of either 1 or 2 genes were selected, and one gene sequence was randomly picked in case of duplication. MUSCLE ${ }^{70}$ was used to build a concatenated sequence alignment. Afterwards IQ-Tree (version 1.7.0b7) ${ }^{71}$ was used to build a concatenated tree using 1,000 bootstraps, estimate the single-locus trees and finally to calculate the gene- and site-concordance factors of the inferred species tree ${ }^{72}$.

\section{HGT detection}

The NCBI non-redundant protein database (download date 08/06/2018) was complemented with the proteomes of 20 species (Table S1). Diamond (version 0.9.18.119) ${ }^{73}$ searches were performed in sensitive mode against this database for all proteins of these 20 species, retaining maximum 1,000 hits per query. Hits were reduced to maximum five sequences for each order and 15 sequences per phylum. Genes families with at least one copy in a diatom and at least one third of the diatom members having a bacterial hit were analysed. The hits of all diatom members were combined and clustered using CDHIT (version 4.6.1) 74 $^{74}$ based on a 95\% identity cut-off. Next, the sequences were aligned with MAFFT (version 7.187$)^{75}$ in automatic mode. Maximum likelihood trees are produced using IQTree (version 1.6.5) ${ }^{71}$ including a test for the best fitting protein model (-mset JTT, LG, WAG, Blosum62, VT, Dayhoff) ${ }^{76}$. The FreeRate model was used to account for rate heterogeneity across sites (-mrate R), empirical base frequencies were calculated (-mfreq $\mathrm{F}$ ) and 1,000 rounds of ultra-parametric bootstrapping (-bb 1000) (UFBoot2) $)^{77}$ were run.

Phylogenetic trees were reordered based on midpoint rooting, unless the whole eukaryotic fraction formed a cluster and then this cluster was used as a subtree for rooting. For every node having a bootstrap support $\geq 90$, and consisting out of a bacterial and eukaryotic subtree the last common ancestor (LCA) was defined. When the eukaryotic subtree consisted out of more than 20 sequences, they could only compose at most $85 \%$ of the total number of sequences belonging to that node. When several nodes complied to these rules having the same eukaryotic fraction, the last common ancestor of the bacterial subset was considered the donor of this event. To avoid classifying endosymbiotic gene transfer (EGT) incorrectly as HGT, only bacterial-to-eukaryotic events were analysed and events older than SAR + Haptophytes, also dubbed SAR+CCTH, were discarded.

\section{Gene family expansion}

Expanded families were delineated by calculating the Z-score profile of the gene copy number per HGT family across all diatoms excluding the allodiploid $F$. solaris. Families where the variance is larger than two and the Z-score for a particular species is larger than three, were deemed expanded in that species.

\section{Contamination detection}


Structural genomic annotation features for sequenced diatoms were retrieved and GC content, coding sequence length, number of introns per gene and intron length were compared between horizontally and vertically transferred genes among several age categories. Also the distance for every gene in $P$. tricornutum to the closest transposable element as defined by ${ }^{27}$, centromeric and telomeric regions elucidated by ${ }^{78}$ was calculated and compared among the different origins. Statistical significance was calculated by the Wilcoxon rank sum test. For the diatoms T. pseudonana and P. tricornutum, whose genomes are resolved on chromosome-scale level, the distribution of HGT genes was plotted using R.

To assess the degree of contamination from Sphingomonas sp. in S. acus, 914 genomes of the order Sphingomonadales were retrieved from NCBI and a nucleotide blast against the $S$. acus genome was performed. Contigs having at least $70 \%$ identity and $25 \%$ alignment coverage were deemed to have Sphingomonadales origin.

\section{Functional interpretation of HGT genes}

The proteomes of all species were functionally annotated using Interproscan (version 60$)^{79}$ in order to obtain functional domain annotations and Gene Ontology (GO) terms. KEGG orthology identifiers ${ }^{80}$ were attained using EggNOG-mapper ${ }^{81}$. For all diatoms, the chloroplast targeting signal was predicted using ASAFind (version 1.1.7) (2. $^{82}$ Only GO terms within the subtree 'biological process' were taken into account. These terms were expanded to also contain all ancestral functional information. GO and Interpro domain enrichment was performed on the HGT genes per species using hypergeometric testing, and multiple hypothesis testing was constrained using Benjamini-Hochberg correction (q value $<0.05)$. Functional enrichments found in at least two species were visualized using the ComplexHeatmap package ${ }^{83}$ ( $R$ version 3.4 ) and clustered using the complete linkage method.

Tandem duplicates were defined as genes belonging to the same gene family and located within 15 genes of each other and identified using i-ADHoRe v3.0 ${ }^{84}$ (alignment method: gg2, gap size 15, tandem gap 15 , cluster gap 15 , q-value 0.85 , probability cut-off 0.01 , anchor_points 3 , level_2_only FALSE, FDR as method for multiple hypothesis correction).

\section{Metatranscriptome analysis}

For several selected HGT gene families involved in cobalamin synthesis a HMM profile was created using hmmer3 v3.1b2 ${ }^{85}$ and these were uploaded to the Ocean Gene Atlas webserver ${ }^{86}$ to query the eukaryotic MATOU gene dataset ${ }^{87}$ (blastp, evalue cut-off $10^{-10}$ ) linked the metatranscriptomic TARA Oceans data. Only sequences taxonomically assigned as diatoms were further analysed. The abundance was estimated as the number of sequence per station and depth.

\section{Population genetics}

Data from ten resequencing strains was downloaded from the public repository SRA (https://www.ncbi.nlm.nih.gov/sra) (SRR6476693-SRR6476702) and these reads were mapped to the Phaeodactylum tricornutum genome using BWA-mem (version 0.7.17) ${ }^{88}$. The read alignments per strain were filtered to only include unique mappings without chimeric alignments using samtools (version 1.6) (99 $^{89}$ SGSGeneLoss (version 0.1) ${ }^{90}$ was run in a relaxed mode to determine to presence/absence pattern of all genes across the ten strains (minCov $=1$, lostCutoff $=0.05$, thus requiring only one read and $5 \%$ gene coverage to be perceived as present). The resulting phylogenetic pattern of HGT genes was visualized using the ComplexHeatmap package ${ }^{83}$ ( $R$ version 3.4 ) and clustered using the complete linkage method.

SNP calling was performed per strain using HaplotypeCaller, after which SNPs were integrated using GenotypeGVCFs. Both methods are available within the GATK framework (version 3.7$)^{91}$. SNPs were 
filtering using the GATK recommended hard filters $(Q D<2.0 ; F S>60.0 ; M Q<40.0 ; M Q R a n k S u m \leq 12.5$; ReadPosRankSum $\leq 8.0)^{92}$ and only bi-allelic SNPs were retained.

To estimate the degree of negative purifying pressure across the proteome, only coding positions having a read depth $\geq 10$ across all strains were considered, calculated using SAMtools mpileup ${ }^{93}$. In total, $89 \%$ of all genic positions could be analysed and 272,235 SNPs were observed in these regions. We used SnpEff (version $4.3 \mathrm{t}$ ) ${ }^{94}$ to predict the individual effect per SNP and $\pi \mathrm{N} / \pi \mathrm{S}$ was calculated taking only the callable positions for complete codons into account and correcting for the allele frequency of the mutation in the population. Statistical significance of difference in selective pressure across mode of inheritance and age classes was calculated by the Wilcoxon rank sum test.

\section{Expression and co-expression analysis}

An expression atlas for every diatom species which has RNA-Seq expression data available was generated. First, relevant experiments were searched using Curse ${ }^{95}$, which also allows the user to identify and curate replicates. The experiments listed in (Table S5) were used to generate the expression compendia. Next, the atlas was generated using Prose ${ }^{95}$, which uses the SRA toolkit to download the raw data locally, FastQC to perform quality control and adapter detection, Trimmomatic for automatic read trimming and finally kallisto for expression quantification in transcripts per million (TPM). Genes were deemed expressed when having a TPM value of at least 3 . The condition-specificity, also known as tau ${ }^{96}$, of every gene was calculated as follows, where $\mathrm{x}$ is the TPM value per condition, max is the maximal expression of a gene and $n$ is the number of conditions in the expression compendium:

$$
\operatorname{tau}=\frac{\sum(1-(x / \max ))}{n}
$$

Condition-specific genes were defined as having a tau value of bigger or equal than 0.9 .

The generated expression atlas for $P$. tricornutum was also used to define co-expression clusters. The Pearson correlation was calculated in a pairwise manner between all genes and the highest reciprocal rank (HRR) $)^{97,98}$ was determined at 23 , by maximizing the recovery of known $\mathrm{GO}$ annotations, while restraining the number of novel predictions. A cluster was defined for every gene based on this cut-off and $\mathrm{GO}$ enrichment using hypergeometric testing was run per cluster. Multiple hypothesis testing was constrained using Benjamini-Hochberg correction (q value $<0.05$ ).

\section{Data availability}

All gene families, phylogenetic trees of horizontal descent and the dating of the HGT events within these gene families are available on Zenodo (https://zenodo.org/record/3555201).

\section{Author contributions}

E.V. wrote the manuscript, performed species topology delineation, HGT detection analysis, functional interpretation, metagenomic and population genomic analysis. T.D. aided in HGT delineation and performed co-expression analysis. C.M.O-C. aided in population genomic analysis and performed expression analysis generation of S. robusta. K.V. supervised the project. All authors read, edited and approved the manuscript.

\section{Acknowledgments}

E.V. wants to acknowledge the funding obtained by the BOF project GOA01G01715. 


\section{References}

1. Dean, P. et al. Transporter gene acquisition and innovation in the evolution of Microsporidia intracellular parasites. Nat Commun 9, 1709 (2018).

2. Gonçalves, C. et al. Evidence for loss and reacquisition of alcoholic fermentation in a fructophilic yeast lineage. elife 7, (2018).

3. Schönknecht, G. et al. Gene Transfer from Bacteria and Archaea Facilitated Evolution of an Extremophilic Eukaryote. Science 339, 1207-1210 (2013).

4. Krasovec, M. et al. Genome Analyses of the Microalga Picochlorum Provide Insights into the Evolution of Thermotolerance in the Green Lineage. Genome Biol Evol 10, 2347-2365 (2018).

5. Ricard, G. et al. Horizontal gene transfer from Bacteria to rumen Ciliates indicates adaptation to their anaerobic, carbohydrates-rich environment. BMC Genomics 7, 22 (2006).

6. Savory, F., Leonard, G. \& Richards, T. The Role of Horizontal Gene Transfer in the Evolution of the Oomycetes. Plos Pathog 11, e1004805 (2015).

7. Bowler, C. et al. The Phaeodactylum genome reveals the evolutionary history of diatom genomes. Nature 456, 239 (2008).

8. Marchetti, A. et al. Ferritin is used for iron storage in bloom-forming marine pennate diatoms. Nature 457, 467 (2008).

9. Husnik, F. \& McCutcheon, J. Functional horizontal gene transfer from bacteria to eukaryotes. Nat Rev Microbiol 16, 67 (2017).

10. Tsaousis, A. D. et al. Evolution of $\mathrm{Fe} / \mathrm{S}$ cluster biogenesis in the anaerobic parasite Blastocystis. Proceedings of the National Academy of Sciences 109, 10426-10431 (2012).

11. Kominek, J. et al. Eukaryotic Acquisition of a Bacterial Operon. 176, 1356-1366.e10 (2019).

12. Stairs, C. W., Roger, A. J. \& Hampl, V. Eukaryotic Pyruvate Formate Lyase and Its Activating Enzyme Were Acquired Laterally from a Firmicute. Mol Biol Evol 28, 2087-2099 (2011).

13. Stairs, C. et al. Microbial eukaryotes have adapted to hypoxia by horizontal acquisitions of a gene involved in rhodoquinone biosynthesis. Elife 7, (2018).

14. Alexander, W. G., Wisecaver, J. H., Rokas, A. \& Hittinger, C. T. Horizontally acquired genes in early-diverging pathogenic fungi enable the use of host nucleosides and nucleotides. Proc Natl Acad Sci USA 113, 4116-4121 (2016).

15. Strese, A.., Backlund, A. \& Alsmark, C. A recently transferred cluster of bacterial genes in Trichomonas vaginalis - lateral gene transfer and the fate of acquired genes. BMC Evol Biol 14, 119 (2014).

16. Chou, S. et al. Transferred interbacterial antagonism genes augment eukaryotic innate immune function. Nature 518, 98 (2015).

17. Harding, T., Roger, A. J. \& Simpson, A. G. Adaptations to High Salt in a Halophilic Protist: Differential Expression and Gene Acquisitions through Duplications and Gene Transfers. Frontiers in Microbiology 8, 944 (2017).

18. Foflonker, F., Mollegard, D., Ong, M., Yoon, H. S. \& Bhattacharya, D. Genomic Analysis of Picochlorum Species Reveals How Microalgae May Adapt to Variable Environments. Mol Biol Evol 35, 2702-2711 (2018).

19. Field, C. B., Behrenfeld, M. J., Randerson, J. T. \& Falkowski, P. G. Primary production of the biosphere: integrating terrestrial and oceanic components. Science 281, 237-240 (1998).

20. Winder, M. \& Cloern, J. E. The annual cycles of phytoplankton biomass. Phil. Trans. R. Soc. B 365, 3215-3226 (2010).

21. Janech, M. G., Krell, A., Mock, T., Kang, J.-S. \& Raymond, J. A. Ice-binding proteins from sea ice diatoms (Bacillariophyceae). J Phycol 42, 410-416 (2006).

22. Nakov, T., Beaulieu, J. \& Alverson, A. Accelerated diversification is related to life history and locomotion in a hyperdiverse lineage of microbial eukaryotes (Diatoms, Bacillariophyta). New Phytol 219, 462-473 (2018).

23. Armbrust, E. et al. The Genome of the Diatom Thalassiosira Pseudonana: Ecology, Evolution, and Metabolism. Science 306, 79-86 (2004). 
24. Lommer, M. et al. Genome and low-iron response of an oceanic diatom adapted to chronic iron limitation. Genome Biol. 13, R66 (2012).

25. Traller, J. C. et al. Genome and methylome of the oleaginous diatom Cyclotella cryptica reveal genetic flexibility toward a high lipid phenotype. Biotechnol Biofuels 9, (2016).

26. Galachyants, Y. et al. Sequencing of the complete genome of an araphid pennate diatom Synedra acus subsp. radians from Lake Baikal. Dokl Biochem Biophys 461, 84-88 (2015).

27. Rastogi, A. et al. Integrative analysis of large scale transcriptome data draws a comprehensive landscape of Phaeodactylum tricornutum genome and evolutionary origin of diatoms. Sci Rep 8, 4834 (2018).

28. Tanaka, T. et al. Oil Accumulation by the Oleaginous Diatom Fistulifera solaris as Revealed by the Genome and Transcriptome. Plant Cell 27, 162-176 (2015).

29. Mock, T. et al. Evolutionary genomics of the cold-adapted diatom Fragilariopsis cylindrus. Nature 541, 536 (2017).

30. Basu, S. et al. Finding a partner in the ocean: molecular and evolutionary bases of the response to sexual cues in a planktonic diatom. New Phytol. 215, 140-156 (2017).

31. Richards, T. \& Monier, A. A tale of two tardigrades. Proc National Acad Sci 113, 4892-4894 (2016).

32. Zakharova, Yu., Adel'shin, R., Parfenova, V., Bedoshvili, Ye. \& Likhoshway, Ye. Taxonomic characterization of the microorganisms associated with the cultivable diatom Synedra acus from Lake Baikal. Microbiology+ 79, 679-687 (2010).

33. Keeling, P. J. \& Palmer, J. D. Horizontal gene transfer in eukaryotic evolution. Nat Rev Genet 9, 605-618 (2008).

34. Eme, L., Gentekaki, E., Curtis, B., Archibald, J. \& Roger, A. Lateral Gene Transfer in the Adaptation of the Anaerobic Parasite Blastocystis to the Gut. Curr Biol 27, 807-820 (2017).

35. Murphy, C. L. et al. Horizontal Gene Transfer as an Indispensable Driver for Evolution of Neocallimastigomycota into a Distinct Gut-Dwelling Fungal Lineage. Appl Environ Microbiol 85, e00988-19 (2019).

36. Vakirlis, N. et al. A Molecular Portrait of De Novo Genes in Yeasts. Mol. Biol. Evol. 35, 631645 (2018).

37. Croft, M. T., Lawrence, A. D., Raux-Deery, E., Warren, M. J. \& Smith, A. G. Algae acquire vitamin B12 through a symbiotic relationship with bacteria. Nature 438, 90-93 (2005).

38. Bertrand, E. M. et al. Phytoplankton-bacterial interactions mediate micronutrient colimitation at the coastal Antarctic sea ice edge. Proc Natl Acad Sci USA 112, 9938-9943 (2015).

39. Heal, K. R. et al. Two distinct pools of $B 12$ analogs reveal community interdependencies in the ocean. Proc Natl Acad Sci USA 114, 364-369 (2017).

40. Helliwell, K. E., Wheeler, G. L., Leptos, K. C., Goldstein, R. E. \& Smith, A. G. Insights into the Evolution of Vitamin B12 Auxotrophy from Sequenced Algal Genomes. Mol Biol Evol 28, 29212933 (2011).

41. Ellis, K. A., Cohen, N. R., Moreno, C. \& Marchetti, A. Cobalamin-independent Methionine Synthase Distribution and Influence on Vitamin B12 Growth Requirements in Marine Diatoms. Protist 168, 32-47 (2017).

42. Helliwell, K. E. et al. Cyanobacteria and Eukaryotic Algae Use Different Chemical Variants of Vitamin B12. Curr. Biol. 26, 999-1008 (2016).

43. Helliwell, K. E. The roles of B vitamins in phytoplankton nutrition: new perspectives and prospects. New Phytol 216, 62-68 (2017).

44. Campbell, G. R. O. et al. Sinorhizobium meliloti bluB is necessary for production of 5,6dimethylbenzimidazole, the lower ligand of B12. Proceedings of the National Academy of Sciences 103, 4634-4639 (2006).

45. Cohen, N. R. et al. Iron and vitamin interactions in marine diatom isolates and natural assemblages of the Northeast Pacific Ocean: Iron and vitamin interactions in diatoms. Limnol. Oceanogr. 62, 2076-2096 (2017). 
46. Cohen, N. R. et al. Iron storage capacities and associated ferritin gene expression among marine diatoms: Iron storage and ferritin expression in diatoms. Limnol. Oceanogr. 63, 1677-1691 (2018).

47. Marchetti, A., Catlett, D., Hopkinson, B., Ellis, K. \& Cassar, N. Marine diatom proteorhodopsins and their potential role in coping with low iron availability. Isme J 9, 2745 (2015).

48. Fortunato, A. E. et al. Diatom Phytochromes Reveal the Existence of Far-Red-Light-Based Sensing in the Ocean. Plant Cell 28, 616-628 (2016).

49. Montsant, A. et al. Identification and comparative genomic analysis of signaling and regulatory components in the diatom Thalassiosira pseudonana. Journal of Phycology 43, 585-604 (2007).

50. Bayer-Giraldi, M. et al. Growth suppression of ice crystal basal face in the presence of a moderate ice-binding protein does not confer hyperactivity. Proc Natl Acad Sci USA 115, 74797484 (2018).

51. Sorhannus, U. Evolution of Antifreeze Protein Genes in the Diatom Genus Fragilariopsis: Evidence for Horizontal Gene Transfer, Gene Duplication and Episodic Diversifying Selection. Evol Bioinform Online 7, EBO.S8321 (2011).

52. Allen, A. et al. Evolution and metabolic significance of the urea cycle in photosynthetic diatoms. Nature 473, 203 (2011).

53. Oh, J. et al. Diatom Allantoin Synthase Provides Structural Insights into Natural Fusion Protein Therapeutics. ACS Chem. Biol. 13, 2237-2246 (2018).

54. Fabris, M. et al. The metabolic blueprint of Phaeodactylum tricornutum reveals a eukaryotic Entner-Doudoroff glycolytic pathway. Plant J 70, 1004-1014 (2012).

55. Allen, A. E. et al. Evolution and Functional Diversification of Fructose Bisphosphate Aldolase Genes in Photosynthetic Marine Diatoms. Mol Biol Evol 29, 367-379 (2012).

56. Whitaker, J. W., McConkey, G. A. \& Westhead, D. R. The transferome of metabolic genes explored: analysis of the horizontal transfer of enzyme encoding genes in unicellular eukaryotes. Genome Biol 10, R36 (2009).

57. Sun, G. \& Huang, J. Horizontally acquired DAP pathway as a unit of self-regulation: Gene transfer and metabolic network. Journal of Evolutionary Biology 24, 587-595 (2011).

58. Jiroutová, K., Horák, A., Bowler, C. \& Oborník, M. Tryptophan Biosynthesis in Stramenopiles: Eukaryotic Winners in the Diatom Complex Chloroplast. J Mol Evol 65, 496-511 (2007).

59. Rastogi, A. et al. A genomics approach reveals the global genetic polymorphism, structure, and functional diversity of ten accessions of the marine model diatom Phaeodactylum tricornutum. ISME J (2019) doi:10.1038/s41396-019-0528-3.

60. Yang, M., Lin, X., Liu, X., Zhang, J. \& Ge, F. Genome annotation of a model diatom Phaeodactylum tricornutum using an integrated proteogenomic pipeline. Mol Plant 11, 1292 1307 (2018).

61. Lehner, B. \& Fraser, A. G. Protein domains enriched in mammalian tissue-specific or widely expressed genes. Trends in Genetics 20, 468-472 (2004).

62. Freilich, S. et al. Relationship between the tissue-specificity of mouse gene expression and the evolutionary origin and function of the proteins. Genome Biol. 6, R56 (2005).

63. Zhang, L. \& Li, W.-H. Mammalian Housekeeping Genes Evolve More Slowly than TissueSpecific Genes. Mol Biol Evol 21, 236-239 (2004).

64. Sideri, T. C., Willetts, S. A. \& Avery, S. V. Methionine sulphoxide reductases protect ironsulphur clusters from oxidative inactivation in yeast. Microbiology 155, 612-623 (2009).

65. Koski, L. B. \& Golding, G. B. The Closest BLAST Hit Is Often Not the Nearest Neighbor. J Mol Evol 52, 540-542 (2001).

66. Philippe, H. et al. Resolving Difficult Phylogenetic Questions: Why More Sequences Are Not Enough. PLOS Biol 9, e1000602 (2011).

67. Olofsson, J. K. et al. Population-Specific Selection on Standing Variation Generated by Lateral Gene Transfers in a Grass. Current Biology 29, 3921-3927.e5 (2019). 
68. Koch, F. et al. The effect of vitamin $B_{12}$ on phytoplankton growth and community structure in the Gulf of Alaska. Limnol. Oceanogr. 56, 1023-1034 (2011).

69. Emms, D. \& Kelly, S. OrthoFinder: solving fundamental biases in whole genome comparisons dramatically improves orthogroup inference accuracy. Genome Biol 16, 157 (2015).

70. Edgar, R. C. MUSCLE: multiple sequence alignment with high accuracy and high throughput. Nucleic Acids Res. 32, 1792-1797 (2004).

71. Nguyen, L.-T., Schmidt, H. A., von Haeseler, A. \& Minh, B. Q. IQ-TREE: a fast and effective stochastic algorithm for estimating maximum-likelihood phylogenies. Mol. Biol. Evol. 32, 268-274 (2015).

72. Minh, B. Q., Hahn, M. \& Lanfear, R. New methods to calculate concordance factors for phylogenomic datasets. http://biorxiv.org/lookup/doi/10.1101/487801 (2018).

73. Buchfink, B., Xie, C. \& Huson, D. H. Fast and sensitive protein alignment using DIAMOND. Nat Methods 12, 59-60 (2015).

74. Fu, L., Niu, B., Zhu, Z., Wu, S. \& Li, W. CD-HIT: accelerated for clustering the next-generation sequencing data. Bioinformatics 28, 3150-3152 (2012).

75. Katoh, K., Misawa, K., Kuma, K. \& Miyata, T. MAFFT: a novel method for rapid multiple sequence alignment based on fast Fourier transform. Nucleic Acids Res. 30, 3059-3066 (2002).

76. Kalyaanamoorthy, S., Minh, B. Q., Wong, T. K. F., von Haeseler, A. \& Jermiin, L. S.

ModelFinder: fast model selection for accurate phylogenetic estimates. Nat Methods 14, 587-589 (2017).

77. Hoang, D. T., Chernomor, O., von Haeseler, A., Minh, B. Q. \& Vinh, L. S. UFBoot2: Improving the Ultrafast Bootstrap Approximation. Mol Biol Evol 35, 518-522 (2018).

78. Diner, R. E. et al. Diatom centromeres suggest a mechanism for nuclear DNA acquisition. Proc Natl Acad Sci USA 114, E6015-E6024 (2017).

79. Jones, P. et al. InterProScan 5: genome-scale protein function classification. Bioinformatics 30, 1236-1240 (2014).

80. Ogata, H. et al. KEGG: Kyoto Encyclopedia of Genes and Genomes. Nucleic Acids Res. 27, 2934 (1999).

81. Huerta-Cepas, J. et al. Fast Genome-Wide Functional Annotation through Orthology Assignment by eggNOG-Mapper. Mol. Biol. Evol. 34, 2115-2122 (2017).

82. Gruber, A., Rocap, G., Kroth, P., Armbrust, E. \& Mock, T. Plastid proteome prediction for diatoms and other algae with secondary plastids of the red lineage. Plant J 81, 519-528 (2015).

83. Gu, Z., Eils, R. \& Schlesner, M. Complex heatmaps reveal patterns and correlations in multidimensional genomic data. Bioinformatics 32, 2847-2849 (2016).

84. Proost, S. et al. i-ADHoRe 3.0-fast and sensitive detection of genomic homology in extremely large data sets. Nucleic Acids Res. 40, e11-e11 (2012).

85. Eddy, S. R. Accelerated Profile HMM Searches. PLoS Comput. Biol. 7, e1002195 (2011).

86. Villar, E. et al. The Ocean Gene Atlas: exploring the biogeography of plankton genes online. Nucleic Acids Res. 46, W289-W295 (2018).

87. Carradec, Q. et al. A global ocean atlas of eukaryotic genes. Nat Commun 9, 373 (2018).

88. Li, H. \& Durbin, R. Fast and accurate short read alignment with Burrows-Wheeler transform. Bioinformatics 25, 1754-1760 (2009).

89. Li, H. et al. The Sequence Alignment/Map format and SAMtools. Bioinformatics 25, 20782079 (2009).

90. Golicz, A. A. et al. Gene loss in the fungal canola pathogen Leptosphaeria maculans. Funct Integr Genomics 15, 189-196 (2015).

91. McKenna, A. et al. The Genome Analysis Toolkit: A MapReduce framework for analyzing nextgeneration DNA sequencing data. Genome Research 20, 1297-1303 (2010).

92. Van der Auwera, G. A. et al. From FastQ Data to High-Confidence Variant Calls: The Genome Analysis Toolkit Best Practices Pipeline. Current Protocols in Bioinformatics 43, (2013). 
93. Li, H. A statistical framework for SNP calling, mutation discovery, association mapping and population genetical parameter estimation from sequencing data. Bioinformatics 27, 2987-2993 (2011).

94. Cingolani, P. et al. A program for annotating and predicting the effects of single nucleotide polymorphisms, SnpEff: SNPs in the genome of Drosophila melanogaster strain $\mathrm{w}^{1118}$; iso-2; iso-3. Fly 6, 80-92 (2012).

95. Vaneechoutte, D. \& Vandepoele, K. Curse: building expression atlases and co-expression networks from public RNA-Seq data. Bioinformatics 35, 2880-2881 (2019).

96. Kryuchkova-Mostacci, N. \& Robinson-Rechavi, M. A benchmark of gene expression tissuespecificity metrics. Brief Bioinform 18, 205-214 (2016).

97. Liesecke, F. et al. Ranking genome-wide correlation measurements improves microarray and RNA-seq based global and targeted co-expression networks. Sci Rep 8, 10885 (2018).

98. Mutwil, M. et al. Assembly of an Interactive Correlation Network for the Arabidopsis Genome Using a Novel Heuristic Clustering Algorithm. Plant Physiol. 152, 29-43 (2010). 


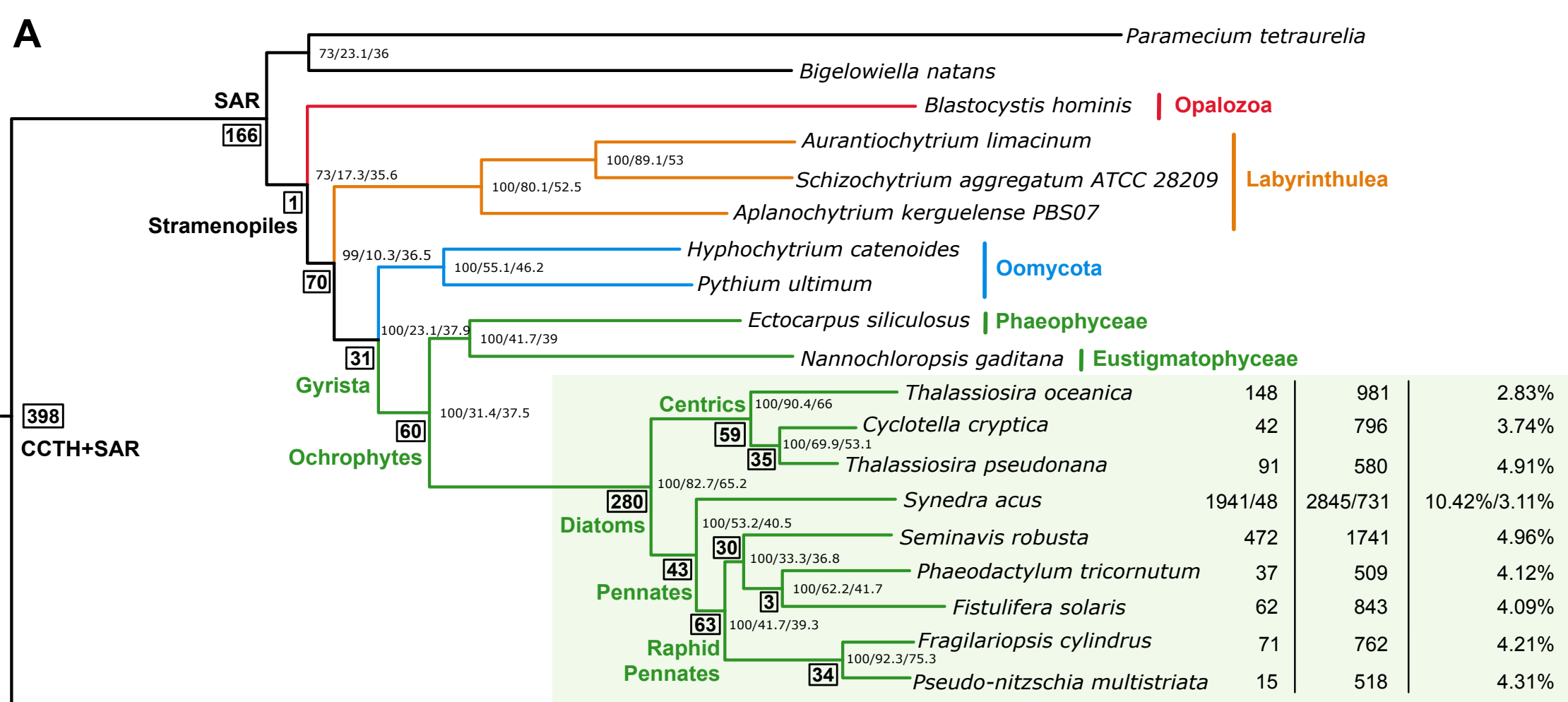

Emiliania huxleyi

Species-specific GFs HGT genes Percentage HGT

B

등 응

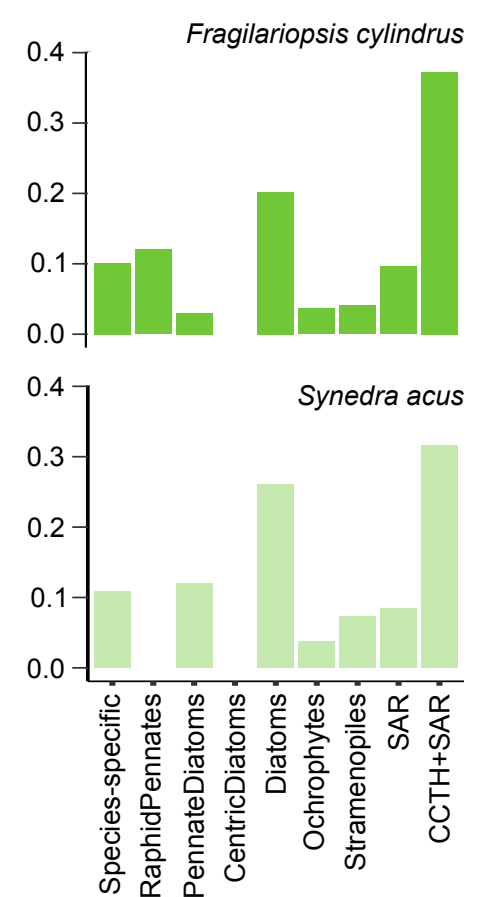

Fistulifera solaris

Pseudo-nitzschia multistriata

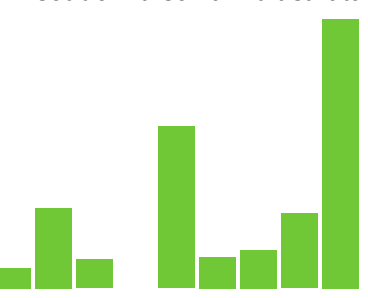

Cyclotella cryptica

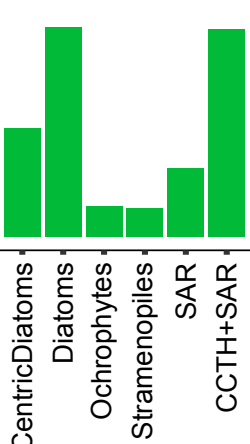

Thalassiosira oceanica

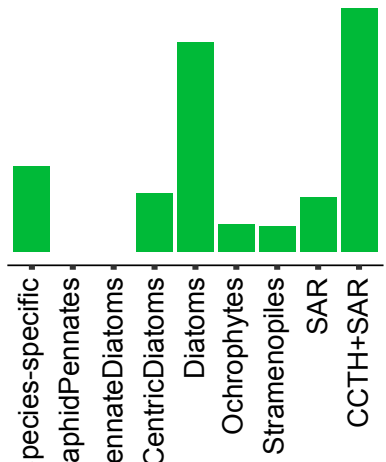

Phaeodactylum tricornutum

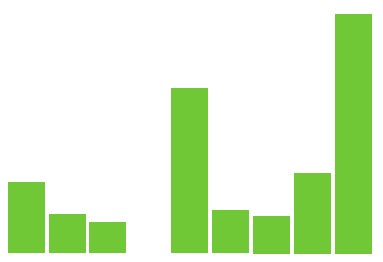

Thalassiosira pseudonana

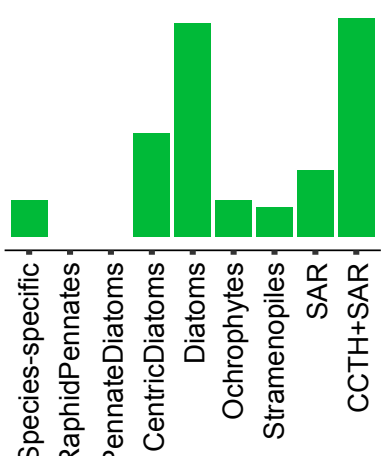

Seminavis robusta

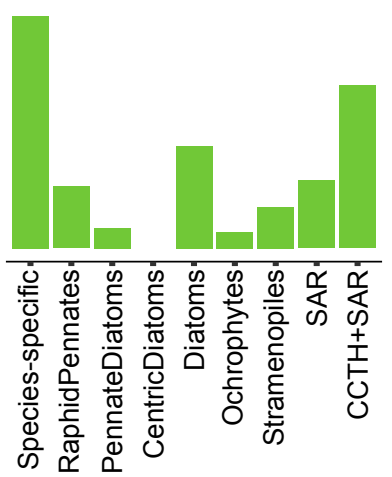

RaphidPennate AraphidPennate PolarCentric 
A Similarity to Sphingomonadales

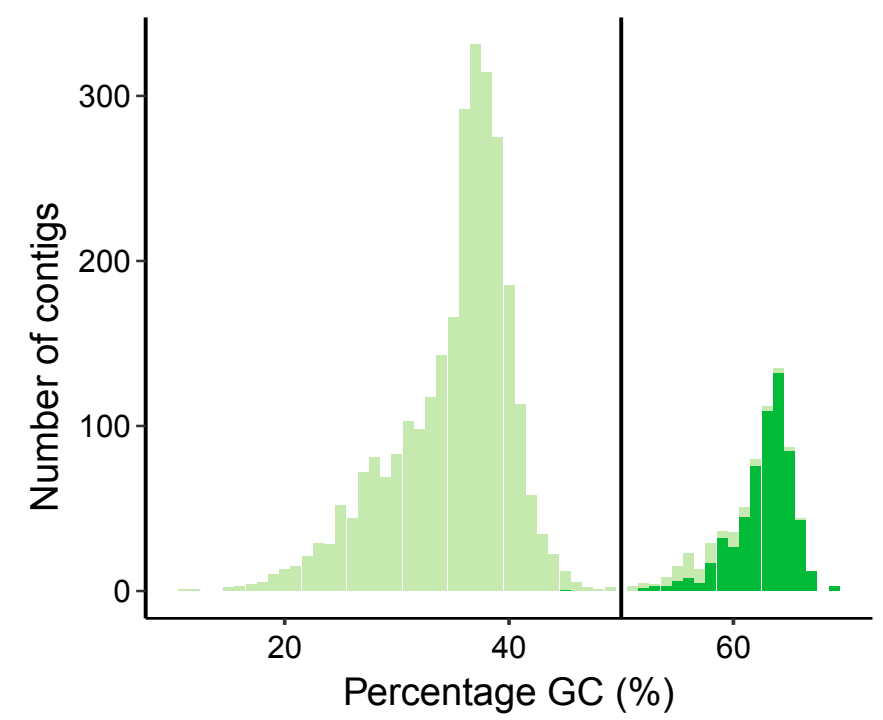

B Presence of a predicted HGT gene

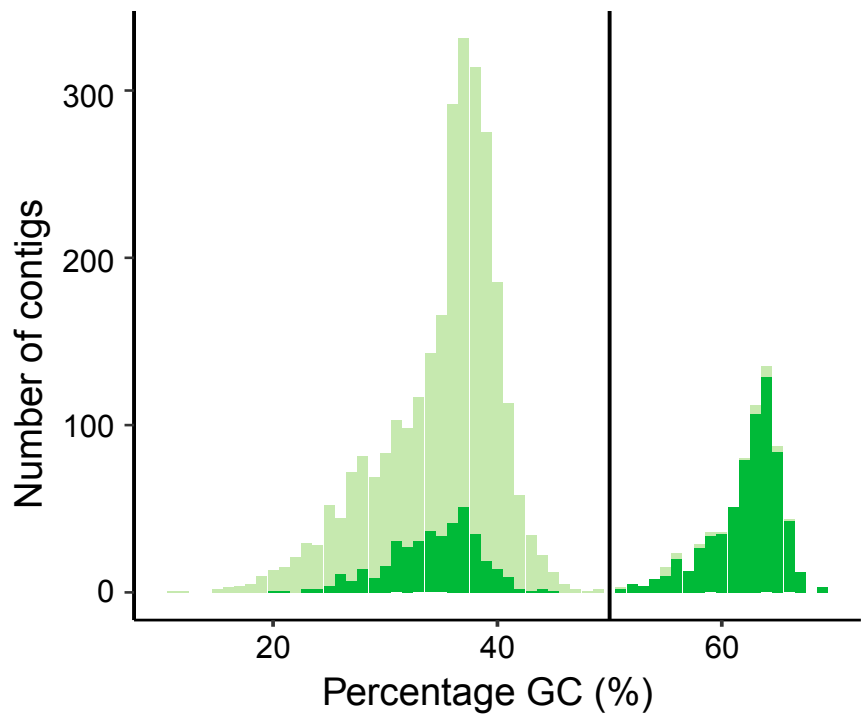


$\stackrel{x}{0}$

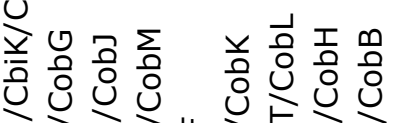

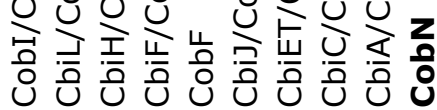

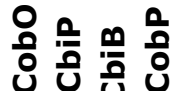

行市

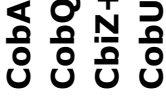

m

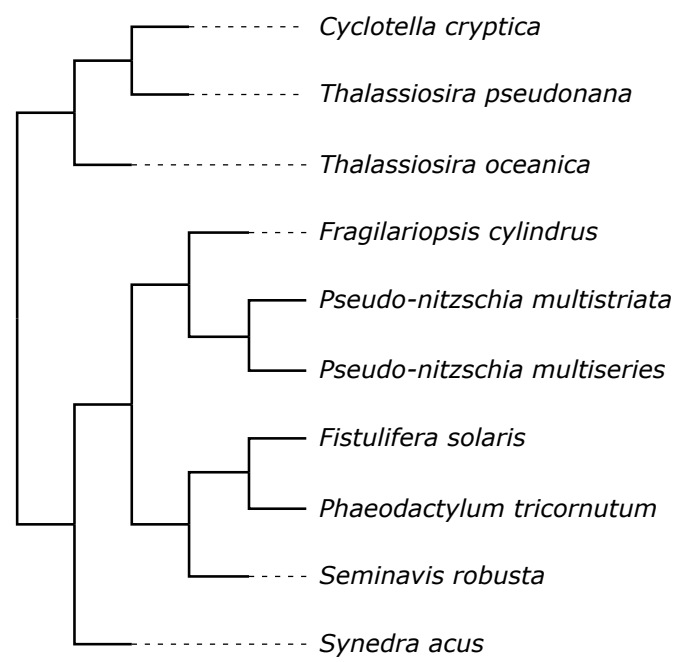

0000000000

$\diamond \diamond \diamond$

$\square \square \square \square$

0000000000

0000000000

000000000

$\diamond \diamond \diamond \diamond$

$\diamond \diamond \diamond \diamond$

$\diamond \diamond \diamond$

$\diamond \diamond \diamond$

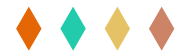

- $\circ \circ \circ \bigcirc \circ \circ \bigcirc \circ$

$\diamond \diamond \diamond \diamond$

$-\circ \circ \circ \circ \circ \circ \circ \circ \circ$

$-\circ \circ \bigcirc \bigcirc \bigcirc \circ \circ \bigcirc \bullet$

$\diamond \diamond \diamond \diamond$

$\diamond \diamond \diamond \diamond$

$\diamond \diamond \diamond \diamond$

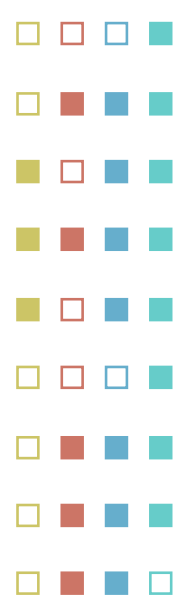

B

CobA

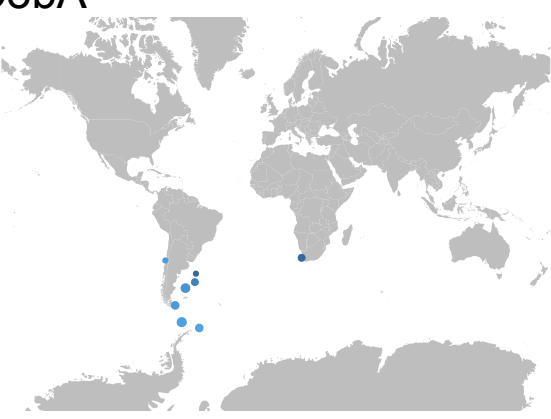

CobU

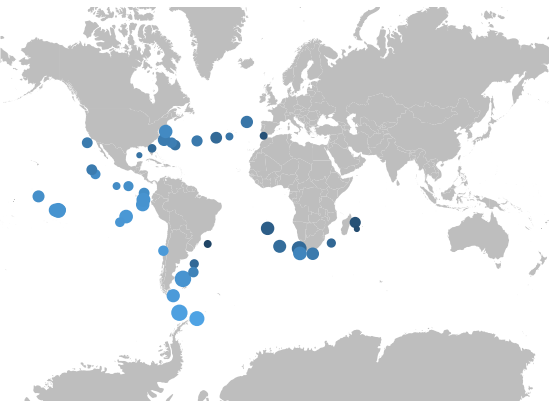

CobQ

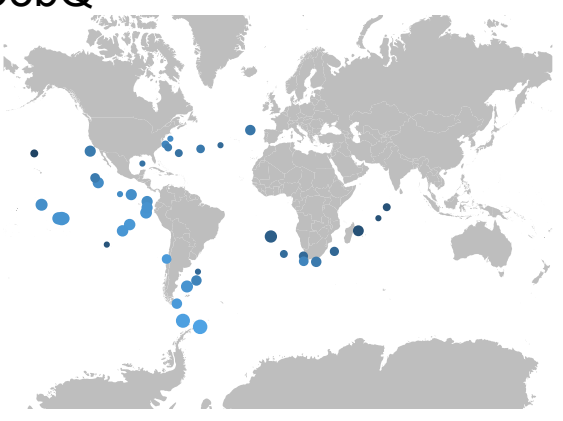

BluB

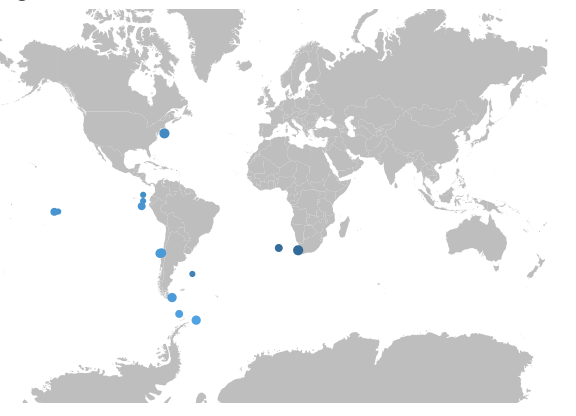

$\mathrm{CbiZ}+\mathrm{CbiB}$

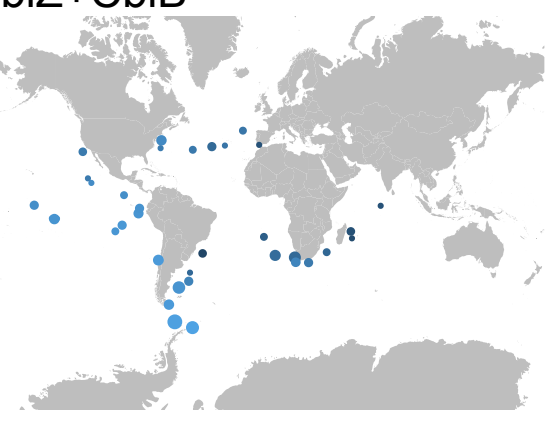

Expression Nitrate ( $\mu \mathrm{M} / \mathrm{L})$

- 1

- 5

- 15

- 40

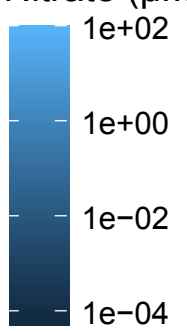




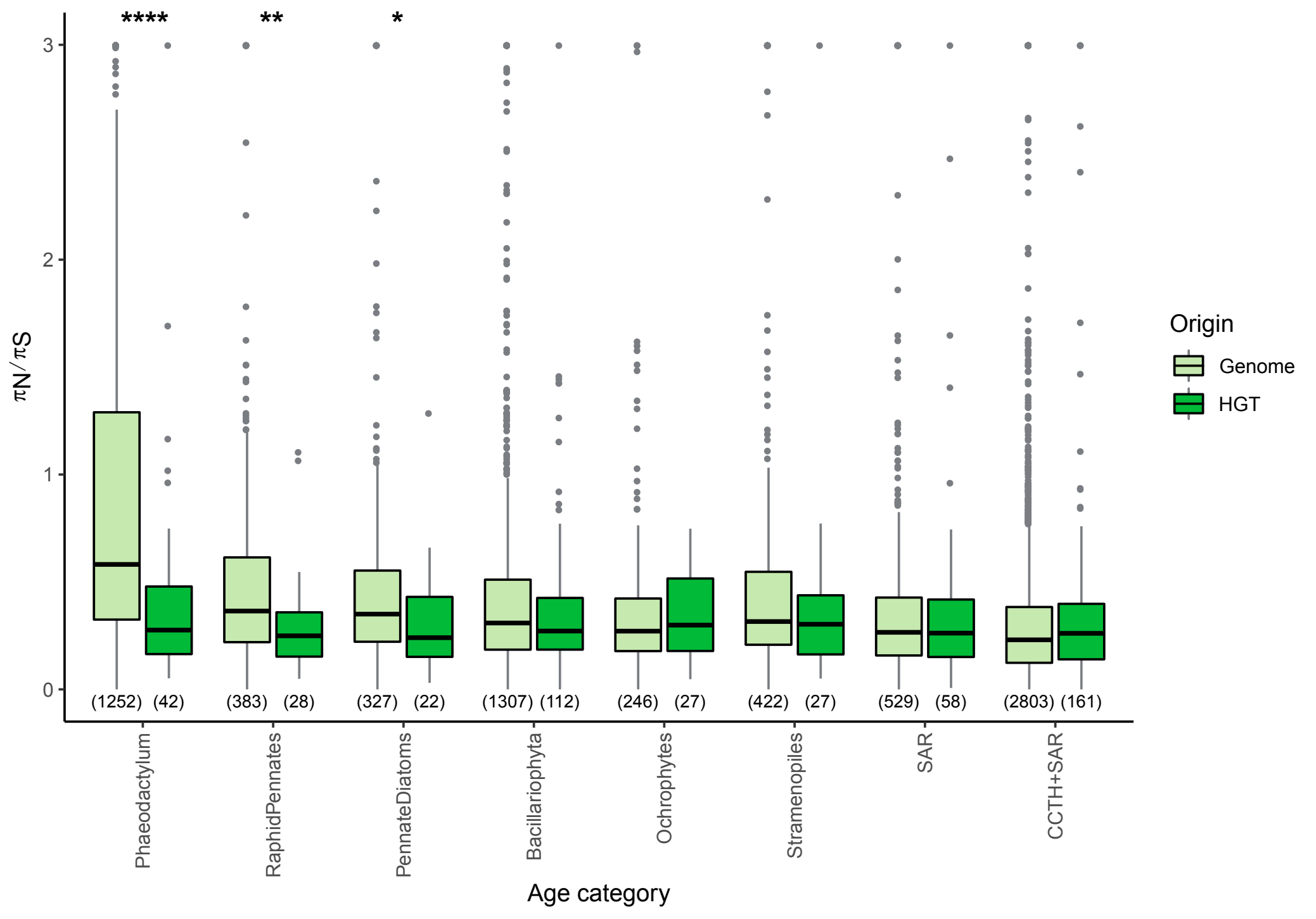


Phaeodactylum tricornutum

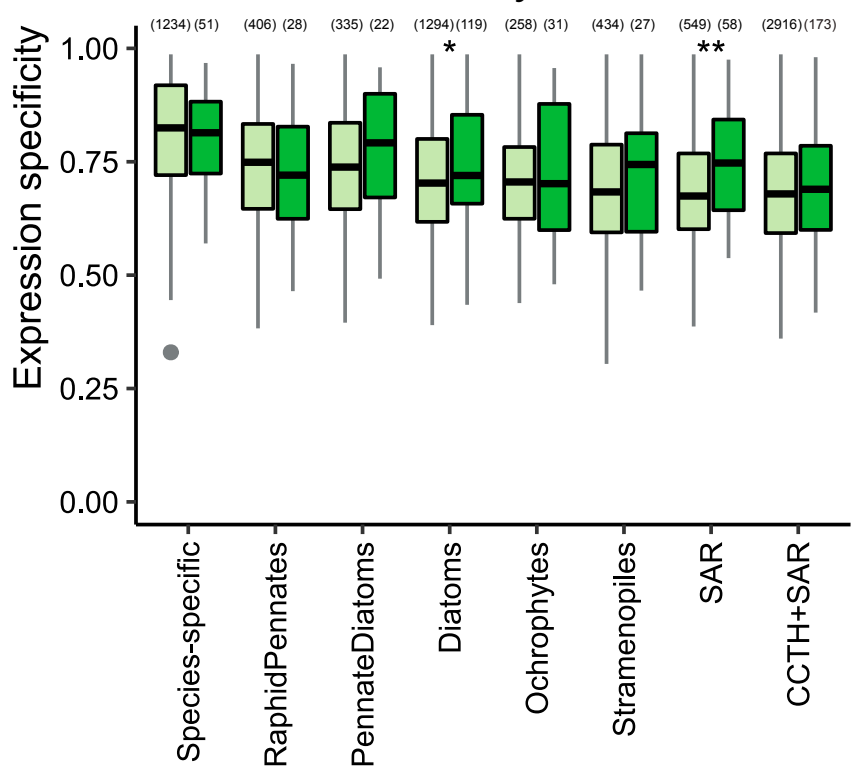

Fragliariopsis cylindrus

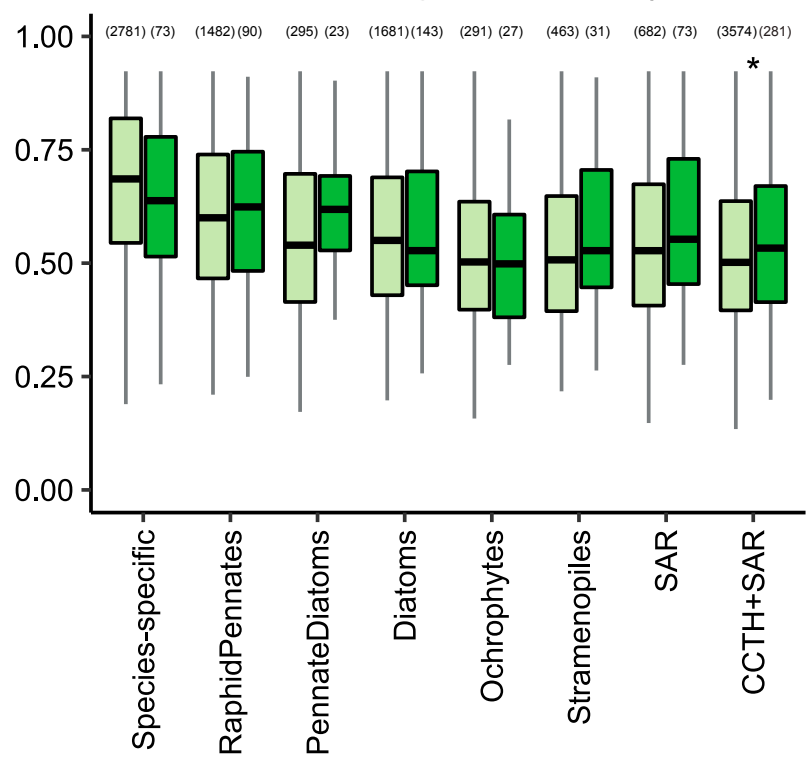

Seminavis robusta

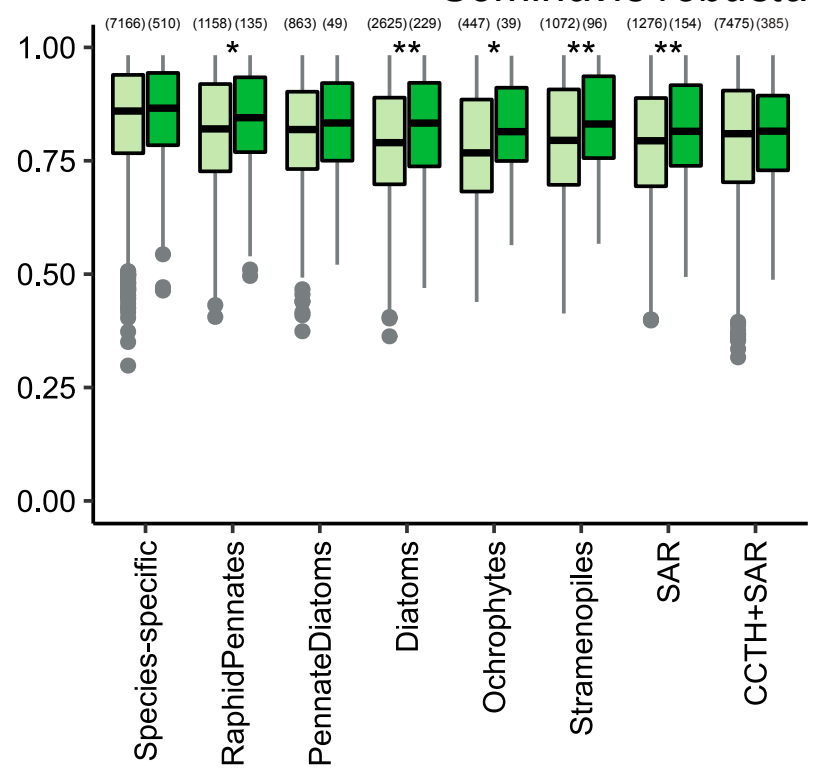

Thalassiosira pseudonana

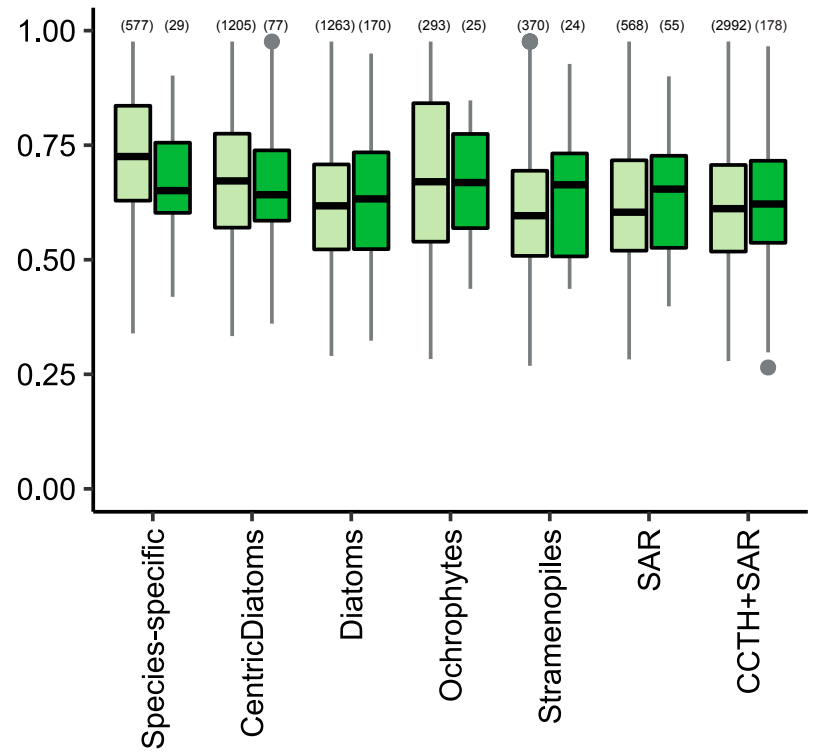

Age category

Origin 由 Genome 追 HGT 